\title{
Venjakob Surface Meeting 2012 in Rheda-Wiedenbrück
}

Ü ber 100 Vertreter der Kunststoffund Automobilzulieferindustrie trafen sich am 25. Oktober 2012 zum 5. Venjakob Surface Meeting in Rheda-Wiedenbrück. Die Teilnehmer folgten der Einladung des Anlagenbauers Venjakob in das A2-Forum und informierten sich über aktuelle Anwendungsbeispiele aus der Praxis. Die An-

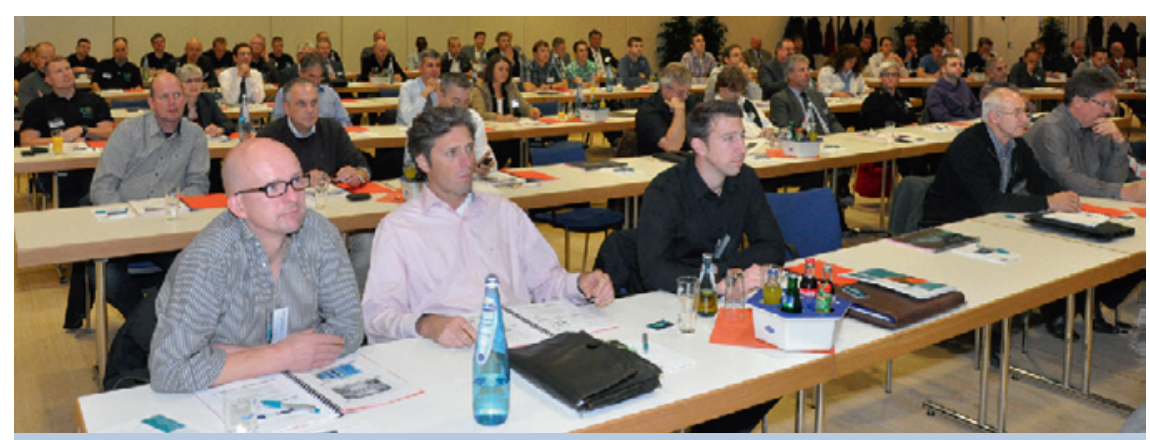

Gut 100 Teilnehmer kamen zum 5. Venjakob Surface Meeting nach Rheda-Wiedenbrück

\section{Krautzberger als "Turnarounder des Jahres" ausgezeichnet}

$D_{s}^{i}$ Krautzberger $\mathrm{GmbH}$ befindet sich nach einer erfolgreich durchgeführten Sanierung wieder auf Wachstumskurs. Damit konnte die existenzbedrohliche Unternehmenskrise, in die der weltweit tätige Spezialist für Oberflächenveredelung Anfang 2009 geriet, überwunden werden. Dem Insolvenzverwalter und Sanierungsexperten, Rechtsanwalt Dr. Jürgen Blersch aus Wiesbaden, ist es gelungen, den Sanierungsplan zusammen mit der Geschäftsführung innerhalb von nur 17 Monaten erfolgreich umzusetzen. Für die Überwindung der Krise und die nachhaltige Sanierung ist die Firma Krautzberger von der Wirtschaftsprüfungsgesellschaft BDO und dem Unternehmermagazin Impulse als "Turnarounder des Jahres" ausgezeichnet worden. Holger Weidmann, geschäftsführender Gesellschafter der Krautzberger $\mathrm{GmbH}$, nahm den Preis am 24. Oktober 2012 in Berlin entgegen. Der Preis, der unter der Schirmherrschaft des Bundeswirtschaftsministers Dr. Philipp Rösler steht, wurde der wendung moderner Beschichtungstechnik stand im Mittelpunkt der eintägigen Veranstaltung. Zum Auftakt gab es einen Ausblick auf die Roboter-Lackiertechnik aus dem Hause Venjakob. Besonderes Interesse galt dem Vortrag über die Vorteile der Lackierung mit reinem Stickstoff anstelle von Pressluft.

Firma Krautzberger in der Kategorie mit bis zu 200 Beschäftigten vergeben.

„Die Chancen einer nachhaltigen Sanierung sind dann am größten, wenn der Insolvenzantrag, wie bei Krautzberger, möglichst frühzeitig von der Geschäftsführung gestellt wird", sagt Sanierungsexperte Blersch. Das sei nicht nur mutig und vorbildhaft, sondern auch verantwortungsvoll den Mitarbeitern und Gläubigern gegenüber. Die Realität zeigt, dass viele Unternehmer den Gang zum Insolvenzgericht erst dann antreten, wenn bereits alle Reserven verbraucht sind und nur noch die Liquidation möglich ist. Im Fall Krautzberger wurde der Turnaround nicht mit Hilfe einer Kapitalzuführung durch einen fremden Investor, sondern durch eine echte Eigensanierung erzielt. Krautzberger erwirtschaftete Erträge und Überschüsse, mit denen sie die Gläubiger befriedigen konnte. Zu Spitzenzeiten beschäftigte Krautzberger bis zu 105 Mitarbeiter. Während der Insolvenz sank die Zahl auf 60 , heute sind 75 Mitarbeiter für das Unternehmen tätig.

\section{Die Lack- und Pulverlackproduktion im 2. Quartal 2012}

S eit Herbst 2011 meldet die deutsche Lackindustrie sinkende Produktionszahlen. Dieser Trend setzte sich auch 2. Quartal 2012 fort. Nach Angaben des Verbandes der deutschen Lack- und Druckfarbenindustrie wurden im 2. Quartal 2012 in Deutschland 207.346 Tonnen Lacke produziert, die ausschließlich oder vorwiegend im Industriebereich eingesetzt werden. Damit sank die Produktionsmenge gegenüber dem Vergleichsquartal im Vorjahr um 4,9\%. Der Produktionswert stieg im gleichen Zeitraum um 1,1 \%, auf rund 729,2 Mio. Euro. Noch stärker ist der Rückgang bei den Pulverlacken. Hier sank die Produktionsmenge um 10,8\% auf 15.242 Tonnen und der Produktionswert ging zurück um $7,6 \%$ auf 70,3 Mio. Euro.

\section{Vollautomatisches Entgrat- und Reinigungszentrum}

Die ATL Anlagentechnik Luhden GmbH und LPW Reinigungssysteme $\mathrm{GmbH}$ haben in einem gemeinsamen Projekt ein neues Entgrat- und Reinigungszentrum für die Johannes Steiner $\mathrm{GmbH} \& \mathrm{Co}$. KG konzipiert und aufgebaut. Das Zentrum ist ausgelegt für das vollautomatische thermische Entgraten, Reinigen und Langzeitkonservieren von Stahl- und Edelstahlteilen. Die Johannes Steiner $\mathrm{GmbH}$ \& Co. KG produziert täglich 1,2 Mio. Überwurfmuttern aus Stahl und Edelstahl für das Common-Rail-System sowie 220.000 Schneidringe und 30.000 Nähmaschinenspulen. Die Realisierung des Projekts dauerte etwa zwölf Monate. Der Fokus des neuen, nach eigenen Angaben weltweit einmaligen Entgrat- und Reinigungszentrums liegt auf der vollautomatisierten Weiterbehandlung der Werkstücke. Täglich werden in einer Schicht durchschnittlich bis drei Tonnen Schüttgut thermisch entgratet und gewaschen. 